Research Paper

\title{
Antioxidant capacity of several Iranian, wild and cultivated strains of the button mushroom
}

\author{
Faezeh Tajalli, Khalil Malekzadeh, Hadi Soltanian, Javad Janpoor, \\ Sharareh Rezaeian, Hamid R. Pourianfar \\ Industrial Fungi Biotechnology Research Department, Research Institute for Industrial Biotechnology, \\ Iranian Academic Centre for Education, Culture and Research, Mashhad, Iran.
}

Submitted: February 25, 2014; Approved: December 19, 2014.

\begin{abstract}
The white button mushroom, Agaricus bisporus, is the most commonly grown mushroom in Iran; however, there is a significant shortage of research on its antioxidant activity and other medicinal properties. The aim of this study was to evaluate antioxidant capacity of the methanolic extracts from four cultivated strains and four Internal Transcribed Spacer (ITS)-identified, Iranian wild isolates of A. bisporus. Evaluations were made for total phenols, flavonoids and anthocyanins, and 2, 2-diphenyl-1-picrylhydrazyl (DPPH) scavenging activity. Overall, results showed that all the wild isolates exhibited significantly lower DPPH-derived $\mathrm{EC}_{50}$, compared to the cultivated strains $(\mathrm{p}<0.05)$. A relatively high relationship was observed between total phenols and flavonoids or anthocyanins $\left(\mathrm{r}^{2}>0.60\right)$. However, these constituents could not statistically differentiate the group of wild samples from the cultivated ones, and there was low correlation with the DPPH-derived $\mathrm{EC}_{50} \mathrm{~s}\left(\mathrm{r}^{2}<0.40\right)$. In conclusion, comparisons showed that wild isolate 4 and cultivated strains A15 and $\mathrm{H} 1$ had higher antioxidant capacity than the others $(\mathrm{p}<0.05)$. This result identifies these mushrooms as good candidates for further investigation.
\end{abstract}

Key words: Agaricus bisporus, antioxidant capacity, wild mushrooms, methanolic extract, anthocyanins.

\section{Introduction}

Reactive oxygen species (ROS) usually refers to both the oxygen-centred radicals of superoxide and hydroxyl and non-radical derivatives of $\mathrm{O}_{2}$ (hydrogen peroxide, singlet oxygen, and hypochlorous acid) (Halliwell, 1999). Overproduction of these reactive agents and/or a lack of effective cellular antioxidant defense, may damage cells through reactions with membrane lipids, nucleic acids, proteins, enzymes or other such small molecules (Sesseira et al., 2009). Consequently, such cellular damage may contribute to many diseases and disorders including several types of cancer, asthma, cardiovascular diseases, diabetes, aging, liver diseases, inflammation, renal failure, cirrhosis, rheumatoid arthritis, arteriosclerosis and neurological disorders (Sesseira et al., 2009; Le Bras et al., 2005).
The cellular antioxidant defense system in the human body generally counteracts oxidative stress through scavenging or minimizing ROS formation using endogenous and/or diet-derived molecules (Halliwell, 2006). Some of the most important molecules involved in this antioxidant defense network include oxidative enzymes, such as superoxide dismutase and catalase and chemical compounds such as alpha-tocopherol, ascorbic acid, carotenoids and polyphenols (Barros et al., 2008). Therefore, it is essential that daily diets include antioxidant-containing foods or supplements in order to assist the antioxidant defence system. In recent years however, many of the synthetic antioxidants added to foods are suspected to be carcinogenic to humans (Yeh et al., 2011). As a result, there has been increasing research done globally to identify natural foods that are rich source of antioxidant such as vegetables, fruits and edible mushrooms.

Send correspondence to H.R. Pourianfar. Industrial Fungi Biotechnology Research Department, Research Institute for Industrial Biotechnology, Iranian Academic Centre for Education, Culture and Research, Mashhad, Iran. E-mail: pourianfar@acecr.ac.ir, pourianfarh@gmail.com. 
Edible mushrooms are well known as excellent sources of protein, fiber, vitamins and minerals. In addition, they possess useful bioactive metabolites with a broad spectrum of pharmacological benefits such as antioxidant activity (Lindequist et al., 2005). Apparently, there is a growing interest in studying chemical and biochemical aspects of antioxidant capacity of cultivated and wild edible mushrooms, many of which have been reviewed by Sesseira et al. (2009). Amongst edible mushroom varieties, the button mushroom (Agaricus bisporus) is still one of the most commonly and widely consumed mushrooms in the world. In addition to those that have been extensively reviewed by Sesseira et al. (2009), a number of recent studies have reported remarkable antioxidant activity for cultivated and/or wild isolates of $A$. bisporus (Abugria and McElhenney, 2013; Barros et al., 2009; Gan et al., 2013; Liu et al., 2013; Ozen and Turkekul, 2010; Sankara-Rao et al., 2013).

In Iran, edible mushrooms have become more popular in recent decades but they are mostly recognized as a good source of protein and other important medicinal values remain largely overlooked. Recently, there have been some limited studies published in Farsi reporting on antioxidant capacity of some Iranian, non-authenticated medicinal mushrooms. However, no research to date has reported antioxidant activity of edible mushrooms, particularly button mushrooms that constitute more than $90 \%$ of the total mushroom production in the country. In addition, there is a considerable shortage of research on nutritional and pharmacological properties of Iranian wild edible mushrooms. This study was done to determine antioxidant activity and antioxidant-associated constituents in methanolic extracts of several cultivated and well-authenticated Iranian wild strains of $A$. bisporus. The wild isolates evaluated in this study are those of $A$. bisporus, and thus the findings may be more applicable as processes of cultivation and spawning have been well standardized for these strains. The findings from this study warrant further research on wild-growing mushrooms as an excellent and natural antioxidant, which can promote health and prevent oxidative-related diseases, particularly cancers.

\section{Materials and Methods}

\section{Mushroom samples}

A total of eight mushroom samples of $A$. bisporus were analyzed. Four strains; A15, U1, H1 and H2, of white button mushrooms that are commercially cultivated in many mushroom farms in Iran, were prepared. In addition, four Iranian wild isolates of $A$. bisporus were collected for the study from various regions of North-eastern Iran during an effort to collect wild $A$. bisporus mushrooms in the year 2012. These samples were morphologically analyzed by specialists in our institute, as well as by molecular authentication using Internal Transcribed Spacer (ITS) sequence analysis. Selection of the wild isolates used for this study was based on ITS-generated genetic distances. Both the cultivated and wild samples were subjected to tissue culture, spawn production and mushroom-growing on compost, based on the procedures adopted at our institute. Fresh fruiting bodies of the resultant mushrooms were then used for methanol extraction.

\section{Molecular identification of the Agaricus species using ITS}

Molecular identification of the wild samples was done using ITS sequence analysis (6). In brief, DNA from pure mycelia culture of each wild mushroom was extracted using the Rapid Fungal Genomic DNA Isolation Kit (FT71415) according to the manufacturer's instructions (Bio Basic Inc.). Two ITS region primers ITS5: GGAAGTAAAAGTCGTAACAAGG and ITS4: TCCTCCGCTTATTGATATGC were used to amplify the following regions in the ribosomal RNA (rRNA) gene: $18 \mathrm{~S}$ rRNA, partial sequence, ITS1, 5.8S rRNA, ITS2, complete sequence, 28S rRNA, and partial sequence (White et al., 1990). PCR assay was then performed with $20 \mathrm{ng}$ of each DNA sample in a total reaction volume of $50 \mu \mathrm{L}$ consisting of the PCR master mix. All non-cloned amplicons were directly sequenced using the primers ITS5 and ITS4 PCR by Macrogen inc. (Korea). The resultant nucleotide sequences were analyzed with the BioEdit Software, version 7.1.3.0 and then searched using BlastN search in GenBank.

\section{Reagents}

Chemicals used for this study were freshly prepared before use; methanol (grade 99.9\%), hydrochloric acid (grade 37\%), acetic acid (grade 99.8\%), chloroform (grade $99 \%$ ), ammonia (grade 25\%), and sulphuric acid (grade 95\%) were all purchased from Merck (Germany). Other chemicals used in the study were gallic acid (Fluka, Cat. no. 54705138.1126284., Spain), 2,2-diphenyl-1-picrylhydrazyl (DPPH) (Sigma-Aldrich, Cat. no. 1898664, Germany), folin-ciocaltue (Panreac, Cat. no. 251567.1609, Spain) and catechin (Fluka, Cat no. 43412, USA). In addition, copper (II) sulfate and ferric chloride (both from Merck, Germany) were kindly gifted from The Research Institute of Food Science and Technology (Mashhad, Iran).

\section{Sample preparation}

Five hundred gram samples of fresh fruiting body from each mushroom were dried at room temperature followed by grinding to a fine powder. Then, $20 \mathrm{~g}$ of the dried material was loaded into soxhlet apparatus (Duran, Germany) and extracted in methanol at $30^{\circ} \mathrm{C}$ for two days. The methanolic extracts were then filtered and concentrated using a rotary evaporator (RE 300, Heidolph, Germany) at $35^{\circ} \mathrm{C}$ to dryness. The dried extracts were stored in the dark c at $4{ }^{\circ} \mathrm{C}$ before use. 


\section{Qualitative determination of antioxidant-related bioactive compounds}

The following myco-constituents were qualitatively measured according to a procedure established in our laboratory that had been adapted from that cited in Adebayo et al. (2012); saponins, alkaloids, flavonoids, tannins, terpenoid, cardiac glycosides, stroids, phlobatanins, and anthraquinones.

\section{Total phenolic content determination}

Total polyphenols of the methanolic mushroom extracts were determined by a modified method of that cited in Mayur et al. (2010) in a 96-well plate, as follows. Firstly, $10 \mu \mathrm{L}$ of six two-fold dilutions of standard gallic acid $(0.05$, $0.1,0.2,0.4,0.8,1.6 \mathrm{mg} / \mathrm{mL}$ ) in methanol were mixed with $100 \mu \mathrm{L}$ of Folin-Ciocalteau 10\%. After three minutes, $100 \mu \mathrm{L}$ of sodium carbonate solution $(7.5 \%)$ was added and the mixture was then topped up with distilled water to one $\mathrm{mL}$. The plates were kept in the dark for $30 \mathrm{~min}$, after which absorbance was read at $750 \mathrm{~nm}$ by Nanodrop apparatus (Epoch-Biotek, USA). Absorbance for gallic acid generated a standard curve with the following equation: $\mathrm{Y}=0.0018 \mathrm{X}-0.0284(\mathrm{R} 2=0.9992)$. The same procedure was carried out with the mushroom extracts at $10 \mathrm{mg} / \mathrm{mL}$. Total phenolic content (TPC) was calculated using the curve equation and expressed as mg of gallic acid equivalents (GAEs) per g of dry weight (dw).

\section{Total anthocyanin determination}

Anthocyanin contents were determined for mushroom samples based on cyanidin-3-glucoside equivalent (CGEs) through a $\mathrm{pH}$ differential method adapted from Lee et al. (2005). In brief, a $0.025 \mathrm{M}$-solution of $\mathrm{KCl}$ was acidified by HCL (to reach $\mathrm{pH}=1$ ), while a $0.4 \mathrm{M}$-solution of $\mathrm{NaCH}_{3} \mathrm{Co}_{2}$ was adjusted to $\mathrm{pH} 4.5$ by $\mathrm{CH}_{3} \mathrm{Co}_{2} \mathrm{H}$. Then, $100 \mu \mathrm{L}$ of each mushroom extract was mixed in methanol (at $10 \mathrm{mg} / \mathrm{mL}$ ) with $900 \mu \mathrm{L}$ of the $\mathrm{KCl}$ solution and the same procedure was performed with the $\mathrm{NaCH}_{3} \mathrm{Co}_{2}$ solution. Amounts of hundred $\mu \mathrm{L}$ of the mixture were then loaded into each well of the 96-well plate. Absorbance was read by the spectrophotometer at two levels; 510 and $700 \mathrm{~nm}$ and calculations were made for total anthocyanins using the following equation $(\mathrm{mg} / \mathrm{L})$ : AxMWxDFx $10^{3} / \varepsilon \times 1$; where $\mathrm{A}=(A 520 \mathrm{~nm}-A 700 \mathrm{~nm}) \mathrm{pH} 1.0-(A 520 \mathrm{~nm}-$ $A 700 \mathrm{~nm}) \mathrm{pH} 4.5$; MW (molecular weight) $=449.2 \mathrm{~g} / \mathrm{mol}$ for cyanidin-3-glucoside; DF = dilution factor; 1 = pathlength in $\mathrm{cm} ; \varepsilon=26900$ molar extinction coefficient, in Lxmolxcm, for cyd-3-glu; and $10^{3}=$ factor for conversion from $\mathrm{g}$ to $\mathrm{mg}$. Final values were then expressed as $\mathrm{mg}$ of CGEs per $100 \mathrm{~g}$ of $\mathrm{dw}$.

\section{Flavonoid determination}

Flavonoids were determined according to a modified method of that cited in Barros et al. (2008). A standard curve was generated using seven two-fold decreasing dilutions of catechin, starting from $2.5 \mathrm{mg} / \mathrm{mL}$. Then, $62.5 \mu \mathrm{L}$ of each dilution was mixed with the following: distilled water $(312.5 \mu \mathrm{L})$ and $\mathrm{NaNO}_{2}$ solution $(5 \%, 18.7 \mu \mathrm{L})$. After five minutes, $\mathrm{AlCl}_{3} . \mathrm{H} 2 \mathrm{O}$ solution $(10 \%, 37.5 \mu \mathrm{L})$ was added. After six minutes, $\mathrm{NaOH}(1 \mathrm{M}, 125 \mu \mathrm{L})$ and distilled water $(68.7 \mu \mathrm{L})$ were added and the solution was mixed well and subjected to spectrophotometry at $510 \mathrm{~nm}$. A standard curve was constructed based on evaluations for absorbance of catechin with the following equation: $\mathrm{Y}=0.055 \mathrm{X}$ $+0.0542(\mathrm{R} 2=0.9884)$. The same procedure was carried out for each mushroom extract at $10 \mathrm{mg} / \mathrm{mL}$. Flavonoid content was measured for each mushroom sample using the curve equation and expressed as mg of catechin equivalent (CEs) per $\mathrm{g}$ of $\mathrm{dw}$.

\section{Scavenging ability on 2, 2-diphenyl-1-picrylhydrazyl $(\mathrm{DPPH})$ radicals}

The micro-titer plate method, established by Arbaayah and Kalsom (2013) was used to evaluate the scavenging ability of mushroom extracts on DPPH radicals. In brief, $50 \mu \mathrm{L}$ of each mushroom extract (twelve decreasing concentrations, starting from $10 \mathrm{mg} / \mathrm{mL}$ ) were mixed with $50 \mu \mathrm{L}$ of a methanolic solution of DPPH reagent $(0.5 \mathrm{mg} / \mathrm{mL})$ and loaded into each well. The same procedure was performed for ascorbic acid as a positive control. In addition, negative controls were made using a mixture composed of $50 \mu \mathrm{L}$ of DPPH and $50 \mu \mathrm{L}$ of methanol per well. Plates were placed in the dark for 30 min. Reduction of the DPPH radical was determined by measuring absorption at $517 \mathrm{~nm}$. Radical scavenging activity was then calculated using the following equation: $\% \mathrm{RSA}=(\mathrm{Ac}-\mathrm{As} / \mathrm{Ac})$ x100; where RSA: radical scavenging activity; Ac: absorbance of the negative control; As: absorbance of the mushroom sample or ascorbic acid. The extract concentration providing $50 \%$ of radical scavenging activity $\left(\mathrm{EC}_{50}\right)$ was calculated by interpolation from the linear regression analysis.

\section{Statistical analysis}

All treatments were applied in triplicate, and each experiment was independently repeated at least two times. Minitab version 16 was utilized to conduct statistical analysis and ANOVA tests. Means were compared using the Tukey method with a significance level ( $\mathrm{p}$ value) of 0.05 .

\section{Results}

\section{Sequence similarity analysis}

In this study, all wild mushroom cultures were purified from DNA that had been isolated and subjected to PCR. Amplification of the ITS1-5.8S-ITS 2 region generated PCR products of $763 \mathrm{bp}$ in size. Blast analysis then revealed that overall sequence similarity between the refer- 
enced strains and the test isolates was greater than $99 \%$ (Table 1), proving that the wild isolates belonged to the $A$. bisporus species.

\section{Determination of qualitative content}

Qualitative chemical tests revealed that all methanolic extracts possessed a variety of important myco- constituents, including flavonoids, saponins, tannins, terpenoids and stroids. However, they showed no detectable amounts of alkaloids, cardiac glycosides, phlobatanins, and anthraquinones. In addition, there was no observable difference in terms of qualitative composition of bioactive compounds between the cultivated strains and the wild isolates.

\section{Radical scavenging activity}

Antioxidant activity of the mushroom isolates was estimated through measuring a decrease in DPPH radical absorption after exposure to the mushroom extracts. In general, results showed that all samples had levels of radical scavenging activity that were dose-dependent, and that the scavenging activity of ascorbic acid (as a positive control) was greater than $90 \%$ for all concentrations (Figure 1). However, some of the mushroom isolates exhibited outstanding amounts of scavenging activity that were not significantly different from those of the positive control $(p>0.05)$. This was particularly prominent with wild iso-

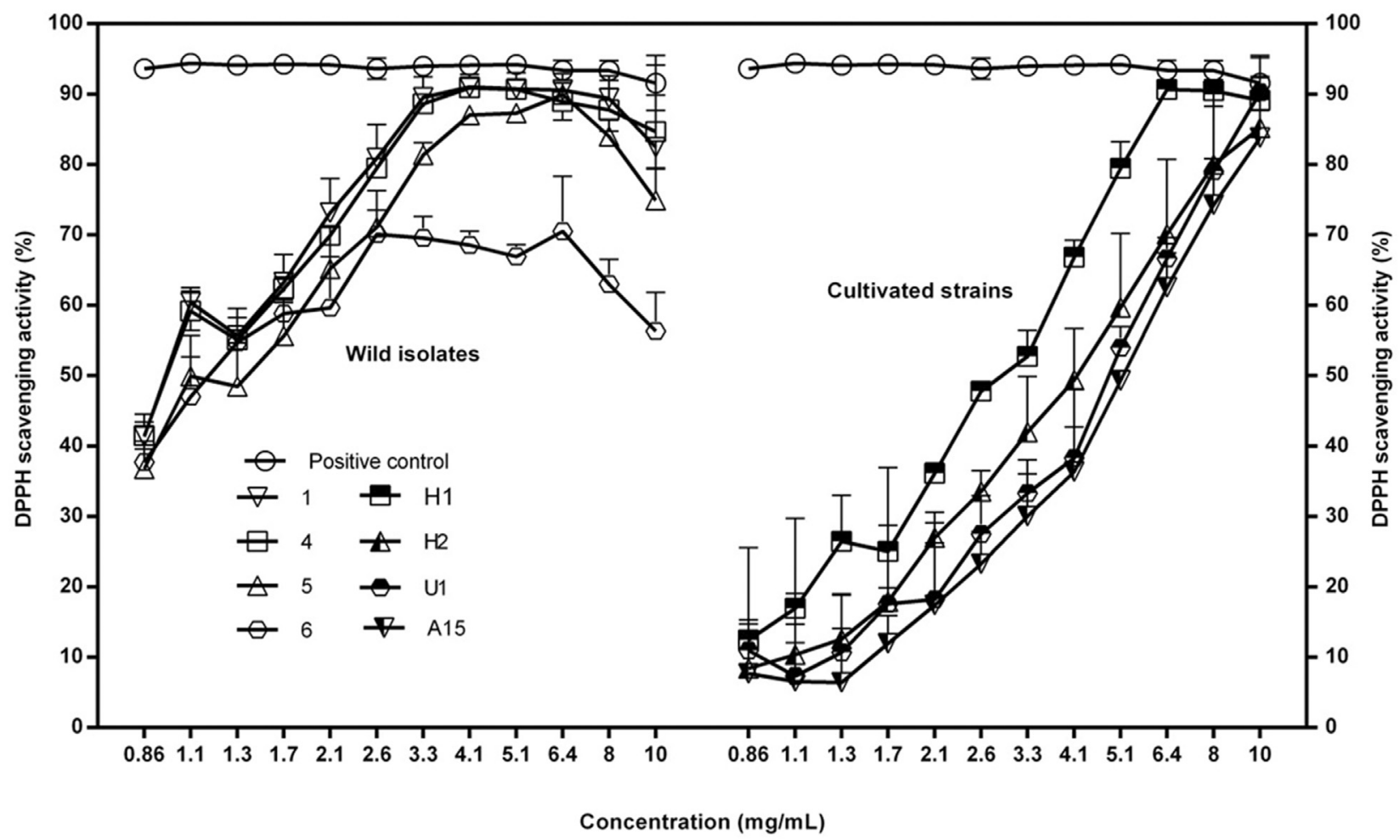

Figure 1 - Dose-response curves of DPPH scavenging activity in wild and cultivated A. bisporus mushrooms. Samples 1, 4, 5, and 6 represent wild-growing isolates of $A$. bisporus, while samples $\mathrm{H} 1, \mathrm{H} 2, \mathrm{U} 1$, and $\mathrm{A} 15$ are commercially cultivated strains of $A$. bisporus. Ascorbic acid was used as a positive control. The mushroom samples and the positive control were tested at 12 dilutions ( 1.25 -fold decreasing concentrations). Values represent reduction of DPPH radicals determined by measuring absorption at $517 \mathrm{~nm}$. Each experiment was independently repeated as least two times and from which standard deviations have been derived.

Table 1 - Blast results of the ITS1-5.8S-ITS 2 region in wild isolates of A. bisporus.

\begin{tabular}{lcccccc}
\hline Sample $^{1}$ & Max score & Total score & Query cover & E value & Identities & $\begin{array}{c}\text { GeneBank Accession number of } \\
\text { referenced strains }\end{array}$ \\
\hline 1 & 1382 & 1382 & $98 \%$ & 0 & $748 / 748(100 \%)$ & HM561977.1 \\
4 & 1376 & 1376 & $98 \%$ & 0 & $747 / 748(99 \%)$ & HM561978.1 \\
5 & 1371 & 1371 & $98 \%$ & 0 & $746 / 748(99 \%)$ & HM561978.1 \\
6 & 1371 & 1371 & $98 \%$ & 0 & $746 / 748(99 \%)$ & HM561977.1 \\
\hline
\end{tabular}

${ }^{1}$ Samples 1, 4, 5, and 6 represent wild-growing isolates of $A$. bisporus. 
lates 1 and 4, which showed remarkable scavenging activity at concentrations above $3270 \mu \mathrm{g} / \mathrm{mL}$ (Figure 1).

In comparison to cultivated strains, the wild isolates exhibited notable scavenging activity even at low concentrations $(\mathrm{p}<0.05)$. In line with the afore-mentioned findings, $\mathrm{EC}_{50}$ values of all the wild isolates were considerably lower than those of the cultivated strains $(\mathrm{p}<0.05)$. Whereas there was no significant difference in $\mathrm{EC}_{50}$ among the wild isolates ( $p>0.05)$, strains $\mathrm{H} 1$ and A15 showed the lowest and the highest $\mathrm{EC}_{50}$ values, respectively, among the cultivated strains $(p<0.05)$ (Table 2). Difference between the wild and cultivated mushrooms, however, decreased in concentrations above $6400 \mu \mathrm{g} / \mathrm{mL}$ (Figure 1).

Statistical comparison was done to compare all of the eight mushroom samples, mean radical scavenging values were calculated for twelve concentrations according to two independent experiments for each sample. These doseindependent results revealed that the wild isolates and the cultivated strains mostly fell into two statistical groups (Figure 2). There were significant differences between wild mushrooms 1, 4 or 5 and all the cultivated strains $(\mathrm{p}<0.05)$. However, there was no meaningful difference among wild mushrooms 1, 4 and 5; and neither was there difference among the cultivated strains $\mathrm{H} 2$, U1, and A15 ( $\mathrm{p}>0.05$ ). Interestingly, wild isolate 6 and cultivated strain $\mathrm{H} 1$ were found to be close to each other without any significant difference $(p>0.05)$. Consequently, there was significant difference between wild isolate 6 and the rest of the wild isolates and also between the cultivated strain $\mathrm{H} 1$ and the other cultivated strains $(\mathrm{p}<0.05)$.

\section{Estimation of total phenolic content}

All the mushroom samples exhibited high amounts of TPC but wild isolate 4 showed an outstanding amount of TPC $(9.6 \pm 0.09 \mathrm{mg}$ GAEs $/ \mathrm{g} \mathrm{dw})$ that was significantly greater than the rest $(p<0.05)$. These findings were compatible with data for radical scavenging, including dose-

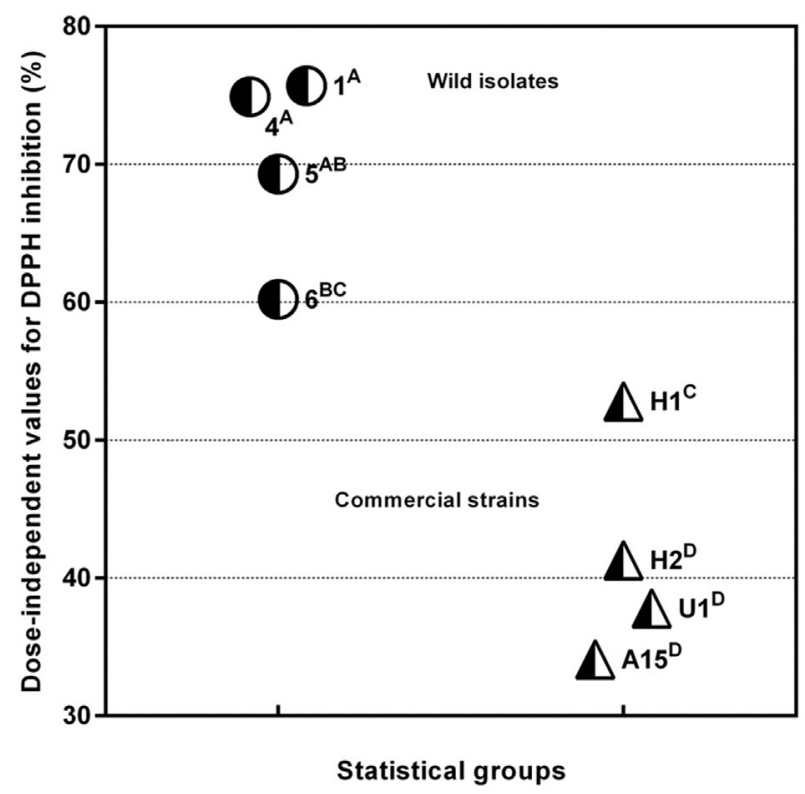

Figure 2 - Statistical comparison of DPPH scavenging activity of A. bisporus mushrooms. Samples 1, 4, 5, and 6 represent wild-growing isolates of A. bisporus, while samples $\mathrm{H} 1, \mathrm{H} 2, \mathrm{U} 1$, and A15 are commercially cultivated strains of A. bisporus. Firstly, analysis of variance for scavenging DPPH by the samples was performed based on all factors, including eight mushroom samples, twelve concentrations and two independent replications. Then, statistical comparison was made between mushroom samples (calculations based on twelve concentrations and two independent replications). Means that do not share a letter are determined as significantly different $(\mathrm{p}<0.05)$.

response curves (Figure 1), $\mathrm{EC}_{50} \mathrm{~s}$ (Table 2), and dose-independent radical scavenging capacity (Figure 2). However, TPC values of some samples contradicted other data on radical scavenging, particularly for the cultivated strain A15 and the wild isolate 5 (Table 2). Whilst the cultivated strain A15 showed a high amount of TPC, the wild isolate 5 showed significantly less $(\mathrm{p}<0.05)$ and was statistically

Table 2 - Comparison of DPPH-derived $\mathrm{EC}_{50}$ values and antioxidant-related compounds between wild and cultivated white button mushrooms.

\begin{tabular}{|c|c|c|c|c|}
\hline Sample $^{1}$ & DPPH-derived $\mathrm{EC}_{50}{ }^{2}(\mathrm{mg} / \mathrm{mL})$ & Total phenols ${ }^{2}$ (mg GAEs/g dw) & Flavonoids ${ }^{2}$ (mg CEs/g dw) & Anthocyanins $^{2}(\mathrm{mg}$ CGEs/100 g dw) \\
\hline 1 & $0.99 \pm 0.15^{\mathrm{a}}$ & $6.13 \pm 0.87^{\mathrm{a}}$ & $3.72 \pm 0.0020^{\mathrm{a}}$ & $4.7 \pm 1.2^{\mathrm{a}}$ \\
\hline 4 & $1.02 \pm 0.05^{\mathrm{a}}$ & $9.61 \pm 0.09^{\mathrm{b}}$ & $4.24 \pm 0.0016^{\mathrm{ab}}$ & $7.7 \pm 0.5^{\mathrm{a}}$ \\
\hline 5 & $1.32 \pm 0.16^{\mathrm{a}}$ & $4.51 \pm 0.22^{\mathrm{c}}$ & $2.78 \pm 0.0004^{\mathrm{d}}$ & $1.13 \pm 0.2^{\mathrm{cd}}$ \\
\hline 6 & $1.26 \pm 0.09^{\mathrm{a}}$ & $8.83 \pm 0.71^{\mathrm{d}}$ & $5.11 \pm 0.0044^{\mathrm{b}}$ & $0.15 \pm 0.01^{\text {ce }}$ \\
\hline H1 & $2.85 \pm 0.18^{\mathrm{b}}$ & $7.67 \pm 0.27^{\mathrm{e}}$ & $3.98 \pm 0.0004^{\mathrm{a}}$ & $1.7 \pm 0.2^{\mathrm{d}}$ \\
\hline $\mathrm{H} 2$ & $4.22 \pm 0.8^{\mathrm{bc}}$ & $4.86 \pm 0.36^{\mathrm{c}}$ & $4.12 \pm 0.0020^{\mathrm{a}}$ & $0.087 \pm 0.1^{\mathrm{e}}$ \\
\hline U1 & $4.82 \pm 0.3^{\mathrm{c}}$ & $3.61 \pm 0.29^{\mathrm{c}}$ & $4.15 \pm 0.0016^{\mathrm{a}}$ & very low ${ }^{3 f}$ \\
\hline A15 & $5.19 \pm 0.07^{\mathrm{c}}$ & $8.81 \pm 0.16^{\mathrm{d}}$ & $4.94 \pm 0.0028^{\mathrm{ab}}$ & $4.6 \pm 0.9^{\mathrm{a}}$ \\
\hline
\end{tabular}

${ }^{1}$ Samples 1, 4, 5, and 6 represent wild-growing isolates of $A$. bisporus, while samples H1, H2, U1, and A15 are commercially cultivated strains of $A$. bisporus.

${ }^{2}$ Values represent mean \pm SD derived from at least two independent experiments. Means that do not share a letter are significantly different $(\mathrm{p}<0.05)$.

${ }^{3}$ Amounts of anthocyanin measured in commercial strain U1 was found to be nearly zero. 
similar to results for cultivated strains $\mathrm{U} 1$ and $\mathrm{H} 2$ $(\mathrm{p}>0.05)$.

\section{Estimation of flavonoid content}

Flavonoid contents among the cultivated strains showed no significant difference ( $\mathrm{p}>0.05$ ), so that all the strains exhibited flavonoid levels of around $4 \mathrm{mg} \mathrm{CEs} / \mathrm{g} \mathrm{dw}$ (Table 2). Among the wild samples, flavonoid contents of isolates 1, 4 and 6 did not show significant difference compared to most of the cultivated strains $(\mathrm{p}>0.05)$. However, isolate 5 showed the smallest amount of flavonoid with $2.78 \mathrm{mg} \mathrm{CEs} / \mathrm{g} \mathrm{dw}$, that was significantly different from the rest $(\mathrm{p}<0.05)$.

\section{Estimation of anthocyanin contents}

Overall, results showed that the trend of anthocyanin values in both wild and cultivated samples were compatible with TPC data, except for that of wild mushroom isolate 6 . Whilst wild isolate 4 possessed the most significant levels of anthocyanin $(7.7 \pm 0.5 \mathrm{mg}$ CGEs/100 g), cultivated strains $\mathrm{H} 2$ and $\mathrm{U} 1$ showed anthocyanin content only in trace amounts (Table 2).

\section{Correlation between the different assays of antioxidant activity}

Pearson's correlation coefficients $\left(\mathrm{r}^{2}\right)$ for the different methods of antioxidant activity conducted in this study were measured. The highest correlation $\left(\mathrm{r}^{2}=0.97\right)$ was observed between the dose-independent radical scavenging capacity (Figure 2) and $\mathrm{EC}_{50}$ values of the DPPH assay (Table 2). A relatively high association $\left(r^{2}>0.60\right)$ was also found between TPC and the content of anthocyanins or flavonoids. However, relationships between DPPH-derived $\mathrm{EC}_{50} \mathrm{~s}$ and $\mathrm{TPC}$, and flavonoids or anthocyanins were low $\left(\mathrm{r}^{2}<0.40\right)$.

\section{Discussion}

This is the first report that compares antioxidant capacity of several ITS-identified Iranian wild mushroom isolates of $A$. bisporus to cultivated strains. A number of studies have investigated the antioxidant potential of wild mushrooms, including Agaricus spp. However, studies have mostly reported antioxidant capability of wild mushrooms and comparison of wild and cultivated mushroom strains has been overlooked (Sesseira et al., 2009). Furthermore, no accurate authentication details have been provided for wild mushroom samples in some of these studies. Differences between mushroom species, method of evaluating antioxidant activity, and the particular solvents utilized for sample extraction, may cause inconsistent results for antioxidant activity (Gan et al., 2013). Thus, our comparisons focused on studies that examined methanolic/ethanol extracts of well-authenticated wild A. bisporus samples.
In our study, all the methanolic extracts exhibited $\mathrm{EC}_{50} \mathrm{~S}$ of around $1 \mathrm{mg} / \mathrm{mL}$, and some of them exhibited radical inhibition of more than $90 \%$ at concentrations over $5 \mathrm{mg} / \mathrm{mL}$. These data represent greater free radical inhibition than methanolic extracts of Turkish wild $A$. bisporus mushrooms, where they were reported to exhibit an $\mathrm{EC}_{50}$ of $19.51 \mathrm{mg} / \mathrm{mL}$ with radical inhibition values of $67 \%$ at $25 \mathrm{mg} / \mathrm{mL}$ (Keles et al., 2011) and $77.5 \%$ at $180 \mathrm{mg} / \mathrm{mL}$ (Elmastas et al., 2007). Our DPPH-derived $\mathrm{EC}_{50}$ values for wild $A$. bisporus isolates are also much lower than those of methanolic extracts of Portuguese wild A. bisporus with $9.61 \mathrm{mg} / \mathrm{mL}$ (Barros et al., 2009). However, an ethanol extract of Chinese A. bisporus showed over $90 \%$ radical inhibition with an $\mathrm{EC}_{50}$ of only $0.38 \mathrm{mg} / \mathrm{mL}$ (Liu et al., 2013).

Among the tested mushrooms, wild isolate 4 and cultivated strain $\mathrm{U} 1$ exhibited the highest and lowest values for phenolic content at 9.6 and 3.5 (mg GAEs/g dry weight), respectively. The maximum TPC observed in wild isolate 4 was lower than that of Turkish wild A. bisporus with $13 \mathrm{mg}$ GAEs/g (Elmastas et al., 2007), and higher than levels of extracts for wild A. bisporus (4.02 mg GAEs/g) from another Turkish study (Keles et al., 2011). The level was also higher than those for wild A. bisporus isolates from Portugal (Barros et al., 2009) and China (Liu et al., 2013) with amounts of 4.49 and $6.18 \mathrm{mg}$ GAEs/g, respectively.

Flavonoid levels were determined as relatively high (about 4 or over, $\mathrm{mg} \mathrm{CEs} / \mathrm{g}$ ) in all cultivated strains as well as wild isolate 4 . Flavonoids belong to phenolic compounds and research has shown antioxidant activity through effectively scavenging many types of ROS (Sesseira et al., 2009). However, there is limited information on flavonoid contents of well-authenticated wild A. bisporus. In comparison to cultivated strains of $A$. bisporus, the findings of this study indicate higher flavonoid contents than those reported for cultivated strains of A. bisporus from Portugal (Barros et al., 2008) and Malaysia (Gan et al., 2013), while flavonoids reported from methanol extracts of white and brown button mushrooms in Tuskegee, Alabama (USA) were significantly higher than those determined in this study (Abugria and McElhenney, 2013).

In this study, anthocyanin levels were determined for the A. bisporus samples, in addition to total phenolic and flavonoid contents. The most significant content of anthocyanins was observed in wild isolates 4 and 5 , and the cultivated strain A15 with determinations of 7.7, 4.7, and 4.6 (mg CGEs/100 g), respectively. Anthocyanins are flavonoids commonly found in fruit and vegetables and research has confirmed their antioxidant activity (He and Giusti, 2010). However, little research has been done to evaluate anthocyanin in relation to antioxidant activity in mushrooms. A study on antioxidant components of the wild medicinal mushroom Boletus edulis (collected from the forests of Gorj) demonstrated presence of anthocyanins at amounts of about 17 and 12 (mg cyanidin chloride/100 g extract) in ethanol and methanolic extracts, respectively. In 
addition, a strong correlation was determined between total anthocyanins and DPPH scavenging activity of the mushrooms (Vamanu and Nita, 2013). Similar results were determined with several Turkish wild edible mushrooms (20-21), where the anthocyanins in mushrooms contributed to antioxidant activity. Thus, high amounts of anthocyanins found in the tested wild mushrooms and the cultivated strain A15 in this study, proposes that anthocyanins are important for conferring antioxidant activity.

The findings of this study showed a low correlation between DPPH-derived $\mathrm{EC}_{50} \mathrm{~S}$ and total phenolic, results that appear to contradict with studies that have reported a significant correlation between DPPH assay and total phenols in mushrooms (Gan et al., 2013; Kim et al., 2008). Such studies suggest that polyphenols are main contributors to antioxidant activity of mushrooms. However, several other studies have shown that total phenols might not necessarily be the only major antioxidant constituent in mushrooms, and as a result low correlation has been determined between total phenols and antioxidant activity, measured by DPPH assays (Barros et al., 2009; Keles et al., 2011; Tsai et al., 2007). In addition, the anthocyanin and flavonoid levels measured in this study had highly correlation with total phenols $\left(\mathrm{r}^{2}>0.60\right)$, which is compatible with the fact that anthocyanins and flavonoids belong to polyphenols.

Among the bioactive compounds qualitatively determined in this study, flavonoids and tannins (as phenolic compounds) have demonstrated antioxidant properties in mushrooms (Sesseira et al., 2009), even though other myco-constituents may also harbor properties with potential therapeutic benefit. In addition, there was no obvious difference in these major myco-constituents between the wild and commercial strains. However, further experiments revealed that wild isolate 4 and cultivated strains A15 and $\mathrm{H} 1$ exhibited greater antioxidant potency compared to the others.

It should be taken into account that wild edible mushrooms may not be consumed directly in the diet because of possible side effects (such as allergies), digestibility, flavor, texture and yield. However, wild mushrooms could be considered as having great potential for the production of useful antioxidant bioactive compounds and as resources for producing drugs (Lindequist et al., 2005). The wild mushrooms used in this study were $A$. bisporus and their cultivation process is very similar to that of cultivated strains of $A$. bisporus. Thus, these mushrooms meet basic requirements necessary in order to recommend further research on their antioxidant-related compounds, where continuous production of large quantity and acceptable quality is needed (Lindequist et al., 2005). Contrary to wild isolates, the cultivated strain A15 is one of the most highyielding and consumer-friendly mushrooms in the Iranian mushroom market; thus its great antioxidant capacity should be of interest for both consumers of mushrooms and for research.

To date, much attention has been paid to the protein content of mushrooms and little has been reported on their antioxidant activity in Iran. Considering the high prevalence of various cancers in the country and the importance of natural antioxidants in cancer prevention, these findings might raise public awareness of mushrooms as having valuable medicinal properties. Although various biochemical and electrochemical experiments are needed to characterize the antioxidant activity of wild mushrooms more precisely, the findings of this study could prompt further research in various fields. Accordingly, it is intended that further study be done to gain a deeper insight into antioxidant activity and phenol profiling of a broader range of Iranian Agaricus species.

\section{Acknowledgments}

This research project was funded by an internal grant to HR Pourianfar from ACECR-Mashhad branch.

\section{References}

Abugria DA, McElhenney WH (2013) Extraction of total phenolic and flavonoids from edible wild and cultivated medicinal mushrooms as affected by different solvents. J Nat Prod Plant Resour 3:37-42.

Adebayo EA, Oloke JK, Ayandele AA et al. (2012) Phytochemical, antioxidant and antimicrobial assay of mushroom metabolite. J Microbiol Biotechnol Res 2:366-374.

Arbaayah HH, Umi Kalsom Y (2013) Antioxidant properties in the oyster mushrooms (Pleurotus spp.) and split gill mushroom (Schizophyllum commune) ethanolic extracts. Mycosphere 4:661-673.

Barros L, Falcão S, Baptista P et al. (2008) Antioxidant activity of Agaricus sp. mushrooms by chemical, biochemical and electrochemical assays. Food Chem 111:61-66.

Barros L, Dueñas M, Ferreira IC et al. (2009) Phenolic acids determination by HPLC-DAD-ESI/MS in sixteen different Portuguese wild mushrooms species. Food Chem Toxicol 47:1076-1079.

Dentinger BTM, Didukh MY, Moncalvo JM (2011) Comparing COI and ITS as DNA barcode markers for mushrooms and allies (Agaricomycotina). PLoS One 6:e25081.

Elmastas M, Isildaka O, Turkekulb I et al. (2007) Determination of antioxidant activity and antioxidant compounds in wild edible mushrooms. J Food Compos Anal 20:337-345.

Gan CH, Nurul Amira B, Asmah, R (2013) Antioxidant analysis of different types of edible mushrooms (Agaricus bisporous and Agaricus brasiliensis). Int Food Res J 20:1095-1102.

Halliwell B (1999) Antioxidant defense mechanisms: From the beginning to the end (of the beginning). Free Radic Res 31:261-272.

Halliwell B (2006) Oxidative stress and neurodegeneration: where are we now? J Neurochem 97:1634-1658.

He J, Giusti MM (2010) Anthocyanins: natural colorants with health-promoting properties. Annu Rev Food Sci Technol 1:163-187. 
Keles A, Koca I, Gençcelep H (2011) Antioxidant properties of wild edible mushrooms. J Food Process Technol 2:6.

Kim MY, Seguin P, Ahn JK et al. (2008) Phenolic compound concentration and antioxidant activities of edible and medicinal mushrooms from Korea. J Agric Food Chem 27:7265-7270.

Le Bras M, Clément MV, Pervaiz S et al. (2005) Reactive oxygen species and the mitochondrial signaling pathway of cell death. Histol Histopathol 20:205-219.

Lee J, Durst RW, Wrolstad RE (2005) Determination of total monomeric anthocyanin pigment content of fruit juices, beverages, natural colorants, and wines by the $\mathrm{pH}$ differential method: collaborative study. J AOAC Int 88:1269-1278.

Lindequist U, Niedermeyer TH, Jülich WD (2005) The pharmacological potential of mushrooms. Evid Based Complement Alternat Med 2:285-299.

Liu J, Jia L, Kan J et al. (2013) In vitro and in vivo antioxidant activity of ethanolic extract of white button mushroom (Agaricus bisporus). Food Chem Toxicol 51:310-316.

Mayur B, Sandesh S, Shruti S et al. (2010) Antioxidant and $\alpha$-glucosidase inhibitory properties of Carpesium abrotanoides L.. J Med Plants Res 4:1547-1553.

Ozen T, Turkekul I (2010) Antioxidant activities of Sarcodon imbricatum wildly grown in the Black Sea region of Turkey. Pharmacogn Mag 6:89-97.

Ozen T, Darcan C, Aktop O et al. (2011) Screening of antioxidant, antimicrobial activities and chemical contents of edible mushrooms wildly grown in the Black Sea region of Turkey. Comb Chem High Throughput Screening 14:72-84.

Sankara-Rao J, Ravi Kumar K, Kartheek Vale V et al. (2013) Bioactive molecules and their anti-oxidant activity of Agaricus bisporus. J Chem Biol Phys Sci 3:1222-1228.

Sesseira ICFR, Barros L, Abreu RMV (2009) Antioxidants in wild mushrooms. Curr Med Chem 16:1543-1560.

Tsai SY, Tsai HL, Mau JL (2007) Antioxidant properties of Agaricus blazei, Agrocybe cylindracea, and Boletus edulis LWT 40:1392-1402.

Vamanu E, Nita S (2013) Antioxidant capacity and the correlation with major phenolic compounds, anthocyanin, and tocopherol content in various extracts from the wild edible $B o$ letus edulis mushroom. Bio Med Res Int 2013:313905.

White TJ, Bruns T, Lee S et al. (1990) Amplification and direct sequencing of fungal ribosomal RNA genes for phylogenetics. In: Innis MA, Gelfand DH, Sninsky JJ et al. eds. PCR Protocols a Guide to Methods and Applications. New York: Academic press, pp 315-322.

Yeh JY, Hsieh LH, Wu KT et al. (2011) Antioxidant properties and antioxidant compounds of various extracts from the edible basidiomycete Grifola frondosa (Maitake). Molecules 16:3197-3211.

Associate Editor: Carlos Pelleschi Taborda

All the content of the journal, except where otherwise noted, is licensed under a Creative Commons License CC BY-NC. 